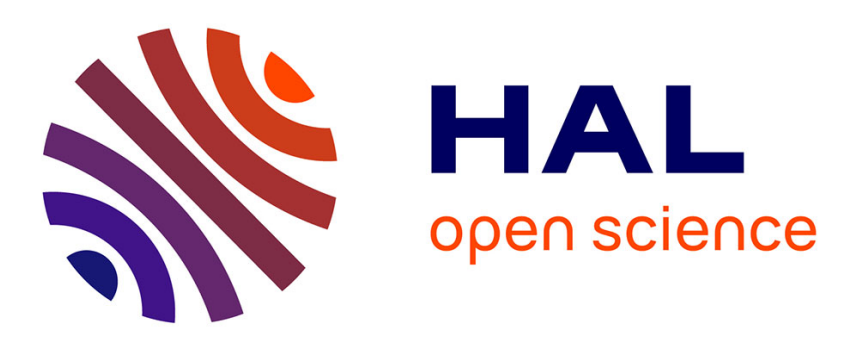

\title{
From discrete to continuous numerical identification of a geomaterial with an internal length
}

\author{
Romain Laniel, Pierre Alart, Stéphane Pagano
}

\section{To cite this version:}

Romain Laniel, Pierre Alart, Stéphane Pagano. From discrete to continuous numerical identification of a geomaterial with an internal length. Computer Methods in Applied Mechanics and Engineering, 2009, 199 (1-4), pp.113-122. 10.1016/j.cma.2009.09.021 . hal-00682650

\section{HAL Id: hal-00682650 \\ https://hal.science/hal-00682650}

Submitted on 26 Mar 2012

HAL is a multi-disciplinary open access archive for the deposit and dissemination of scientific research documents, whether they are published or not. The documents may come from teaching and research institutions in France or abroad, or from public or private research centers.
L'archive ouverte pluridisciplinaire HAL, est destinée au dépôt et à la diffusion de documents scientifiques de niveau recherche, publiés ou non, émanant des établissements d'enseignement et de recherche français ou étrangers, des laboratoires publics ou privés. 


\title{
From discrete to continuous numerical identification of a geomaterial with an internal length
}

\author{
Romain Laniel ${ }^{\mathrm{a}, *}$ Pierre Alart ${ }^{\mathrm{b}}$ Stéphane Pagano ${ }^{\mathrm{b}}$ \\ ${ }^{\mathrm{a}}$ LARMAUR, Campus de Beaulieu Bât. 10B 35042 Rennes cedex, France \\ ${ }^{\mathrm{b}}$ LMGC, Universite Montpellier 2, Place Eugène Bataillon, cc 048, 34095 \\ Montpellier cedex 5, France
}

\begin{abstract}
A geomaterial called TexSol and composed of sand and wires was investigated by numerical experiments in order to determine its geometrical and mechanical parameters, such as tortuousness of the wire, anisotropy and characteristic length. This stage is essential for studying a material with an obvious non-local behavior. Investigations by discrete elements highlighted that the characteristic length was dependant on the loading level. These simulations provided access to variables that standard physical experiments cannot provide. Some parameters of a continuous model of TexSol were identified through discrete numerical experiments using a classic procedure. The other parameters were determined by finite element method updating.
\end{abstract}

Key words: characteristic length, unilateral reinforcement, numerical homogenization

\section{Introduction}

Civil engineers have proposed many different solutions (geogrid, columns, geomembranes et catera) to enhance the mechanical features of geomaterials. One original solution is to insert very long wires into the sand. These are distributed randomly, thus creating a very tortuous and tangled reinforcement network wedged between the sand grains. A TexSol bank is made layer by layer

* e-mail: romain.laniel@univ-rennes1.fr phone: +332.23 .23 .56 .10$ 
by projecting both sand grains and continuous wires on the free surface of the bank. This geomaterial and wire mixture, as represented in Fig. 1, is called TexSol and was created in 1984 by Leflaive, Khay and Blivet of the French Laboratoire Central des Ponts et Chaussées [5, 9]. This process increases the

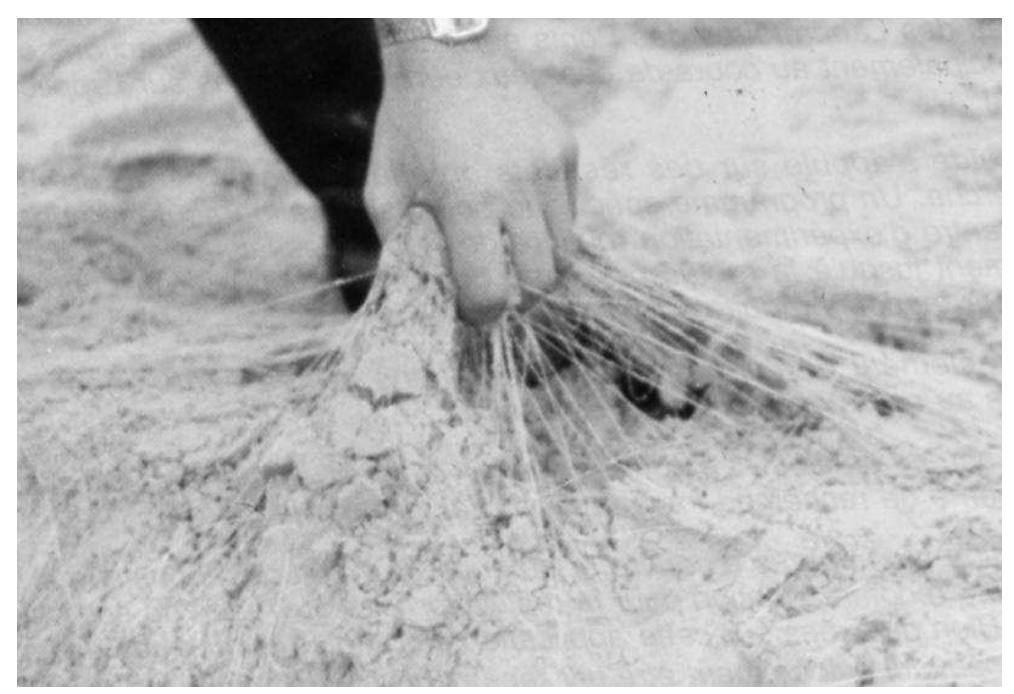

Fig. 1. TexSol bank illustration elicit from : M. Khay and J-P. Gigan. TEXSOL Ouvrage de soutènement. $L C P C, 1990$.

bearing pressure of the ground by distributing the dominating load over a wider area. Thanks to their compactness and flexibility, wires can be intercalated between the sand particles without modifying the granular structure. However, when the material is loaded, the wires may come into contact due to sand particle displacement, thus including particular interactions between the two phases. Several reinforcement mechanisms are assumed to be involved, such as direct wire tension between two blockings, the friction of a tended wire segment strongly curved on sand, or fiber-crossing. The scope of the present TexSol study does not encompass optimization of a law of engineering behavior. LCPC in Rouen (France) carried out experiments and obtained empirical data on TexSol behavior on the basis of which it then drew up construction and usage guidelines. However, there are still many basic unanswered questions concerning this particular composite material. Interactions between the two phases, which are sensitive to the random distribution of the wire, are complex and hard to clarify. On the other hand, in the absence of physicochemical cohesion interactions between its elements, it is the chaotic nature of the wire network that ensures the overall cohesion of the material. The unique feature of the TexSol is that it combines a low interaction length geomaterial with a "structure" in weak volume fraction but with a very large interaction length. Discrete numerical investigations are effective for revealing the non-local or local properties of TexSol in a range of mechanical tests. Indeed, the Non-Smooth Contact Dynamics method simulates mechanical tests on granular samples. This provides new access to state variables such as the positions and velocities of all the particles as well as the contact reaction net- 
work, or continuous variables such as stress and strain per phase. In addition, a macroscopic continuous law, dedicated to local reinforcement, should be used to make a homogenization attempt at comparing a continuous and discrete simulation.

\section{First microstrucural characterizations of numerical samples}

In this section, reinforcement mechanisms involving long or short interaction length, i.e. characteristic length, are studied using a range of discrete simulations from quasi-static to dynamic or small to large transformations.

\subsection{TexSol discrete model}

The NSCD is a discrete element method used in the $L M G C 90$ code which simulates multibody vs. multicontact problems, while favoring velocity fields $[4,10]$. For a rigid body collection with $q$ and $R$, the Lagrange coordinate vector and the contact reactions and torques vector, respectively, the dynamic equations are expressed as $M \ddot{q}(t)=F_{\text {ext }}(t)+R$, where $F_{\text {ext }}$ and $M$ are the external forces and mass matrix, respectively. The NSCD integration scheme is a $\theta$-method with $\theta$ ranging between 0.5 and 1 . The velocity, free of contacts is defined as $\dot{q}_{\text {free. }}$. For a single contact $\alpha$ problem, NSCD evaluates the external forces and dynamic effects on the contactor point. To make such a transformation, $H_{\alpha}$ and $H_{\alpha}^{T}$ are used to move variables from the local contact frame to the global body and vice-versa. The local contact $\alpha$ variables $u^{\alpha}$ and $r^{\alpha}$, i.e. the relative velocity and the contact reactions in the contact local frame, respectively, are defined with $u^{\alpha}=H_{\alpha}^{T} \dot{q}$ and $R=H_{\alpha} r^{\alpha}$. We also introduce the average impulse $p^{\alpha}=\int_{t_{i}}^{t_{i+1}} r^{\alpha} \mathrm{d} t$ and can write

$$
\left\{\begin{array}{l}
u_{i+1}^{\alpha}-W^{\alpha \alpha} p_{i+1}^{\alpha}=u_{\text {free }, i}^{\alpha}+\sum_{\beta \neq \alpha} W^{\alpha \beta} p_{i+1}^{\beta} \\
\operatorname{Law}\left[u_{i+1}^{\alpha}, p_{i+1}^{\alpha}\right]=\text { true }
\end{array}\right.
$$

The smooth dynamic effects are included in the expression of relative free velocity $u_{\text {free }, i}^{\alpha}$. The Delassus operator $W^{\alpha \beta}=H_{\alpha}^{T} M^{-1} H_{\beta}$ naturally appears in the dynamics reduced to contacts. In this way, for a frictionless problem with a Signorini contact condition, system (1) proves to be a standard Linear Complementarity Problem,

$$
\left\{\begin{array}{l}
u_{i+1}-W p_{i+1}=u_{\text {free }, i} \\
0 \leq u_{i+1} \perp p_{i+1} \geq 0
\end{array}\right.
$$



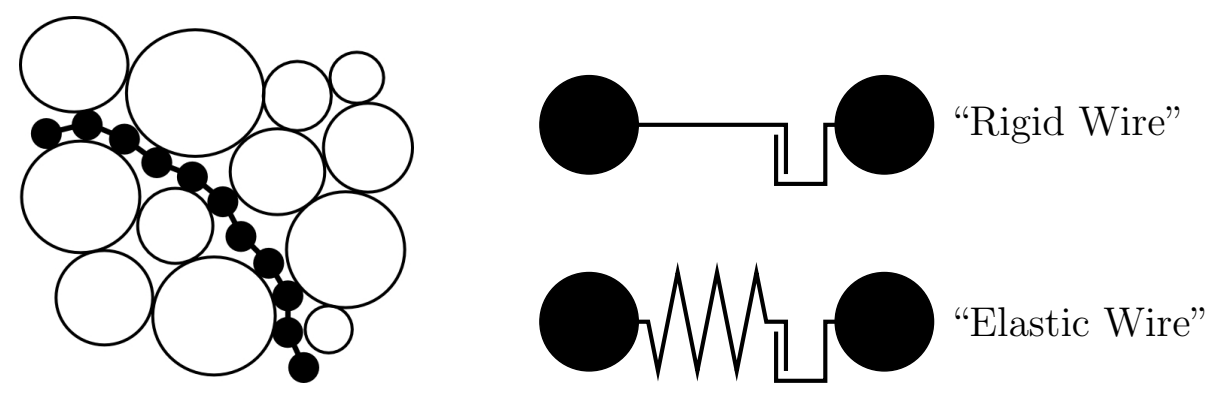

Fig. 2. Illustrations of a two-dimensional TexSol sample (right) and of specific interaction laws used to model the wire (left). The chain of beads is shown in black and is trapped between white sand particles.

For a frictional contact problem, tangential reactions and tangential velocities have to verify a similar non-smooth relation. A Gauss - Seidel loop computes all contact reactions until convergence. Moreover, in a quasi-static pattern, shocks can be considered as inelastic [4]. As TexSol is a reinforced granular material, the sand phase can be perfectly integrated in this model. However, this is not the case for the reinforcement network. A discrete model of wires using a chain of beads (cf. Fig. 2) and specific interactions is proposed in [7]. On one hand, beads interact with other spherical sand particles via a classic Signorini - Coulomb contact law. On the other hand, their centers are connected by unilateral interactions. Variable changes $\widetilde{u}_{n}=\frac{g_{\mathrm{ref}}-g^{-}}{h}-u_{n}$ and $\tilde{p}_{n}=-p_{n}[2]$ are used to define these laws, where $n$ is the index of contact normal component, $g^{-}=g\left(t_{i}\right)$ is the minimum distance between two particles at the beginning of the considered time step and $g_{\text {ref }}=\frac{g(0)}{1+\varepsilon_{0}}$ is the gap with no force and no displacement on the contact element ( $\varepsilon_{0}$ is the prestrain). They can be rigid or elastic and at the same time, unilateral or bilateral. Indeed, we showed in [8] that bilateral wire interactions give unrealistic phenomena. The only two unilateral interactions are:

- "Rigid wire": $u_{n} \leq \frac{g_{\mathrm{ref}}-g^{-}}{h}, p_{n} \leq 0, u_{n} p_{n}=0$. With variables $\widetilde{u}_{n}$ and $\widetilde{p}_{n}$ a classical complementarity relation is obtained,

$$
0 \leq \widetilde{u}_{n} \perp \widetilde{p}_{n} \geq 0
$$

- "Elastic wire": this includes the wire stiffness parameter $k$,

$$
p_{n}=\left\{\begin{array}{ll}
0 & \text { if } u_{n} \leq \frac{g_{\mathrm{ref}}-g^{-}}{h} \\
-h^{2} k\left(u_{n}-\frac{g_{\mathrm{ref}}-g^{-}}{h}\right) & \text { if } u_{n} \geq \frac{g_{\mathrm{ref}}-g^{-}}{h}
\end{array} .\right.
$$

If we consider another variable change, $\widehat{u}_{n}=\frac{g_{\mathrm{ref}}-g^{-}}{h}-u_{n}-\frac{p_{n}}{h^{2} k}$, the previous condition can be written as a complementarity relation,

$$
0 \leq \widehat{u}_{n} \perp \widetilde{p}_{n} \geq 0
$$


The discrete wire model was checked with both laws using several tests: geometrical, vibrating, woven or tensegrity structure behavior et catera.

\subsection{Impact test}

Mechanical tests can be carried out once the TexSol sample clearly generates as a granular media using a tailored preprocessor. The first, a crash test on a TexSol slope, is a highly dynamic test implementing several reinforcement mechanisms and allowing discrete model relevancy to be checked. Indeed, a spherical $\varnothing 1.4 \mathrm{~mm}$ diameter impactor, is projected, at $10 \mathrm{~m} . \mathrm{s}^{-1}$, at a sand slope and a TexSol slope. Both slopes are generated by a numerical granular sample deposited on a rubber surface. They consist of 37000 particles with polydispersity ranging from $\varnothing 0.2 \mathrm{~mm}$ to $\varnothing 0.6 \mathrm{~mm}$. The TexSol sample includes 6000 beads of $\varnothing 0.2 \mathrm{~mm}$ diameter to model a wire network of $500 \mathrm{~km} . \mathrm{m}^{-3}$ volume length density which is quasi-equiprobably distributed. The tracks in wire-reinforced geomaterial are shallower and more superficial

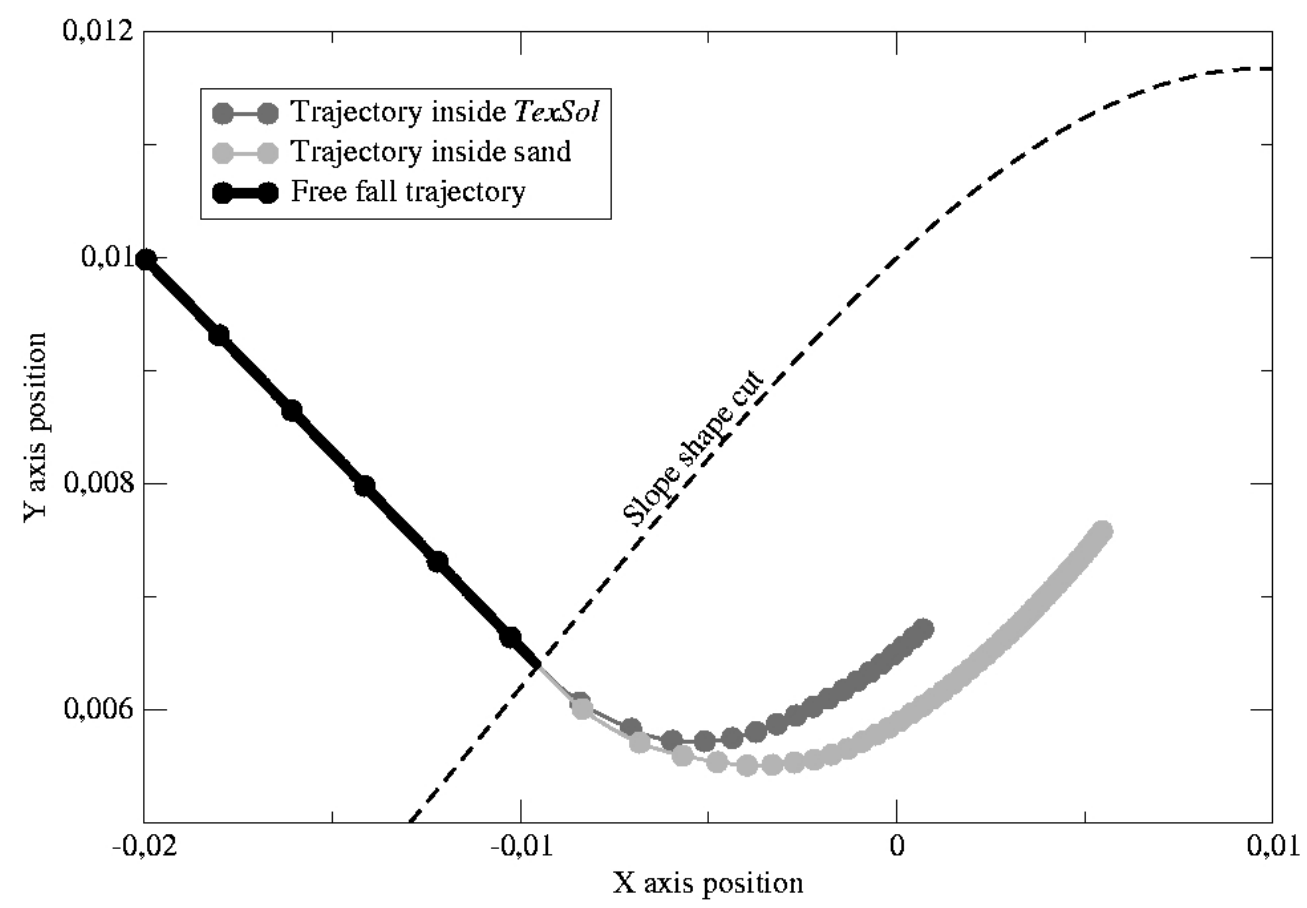

Fig. 3. Trajectory plots of an impactor through a sand slope (light grey) and through a TexSol slope (dark grey); each plot is dotted every $0.2 \mathrm{~ms}$. The free surface of slopes, cut in the trajectory plan, is represented by a dashed line.

than in sand (cf. Fig. 3). In both cases, the trajectories are deflected toward the top of the pile because of the greater level of the force network at the bottom [11]. Moreover, impactor deceleration is greater in the TexSol because each wire hit by the projectile impedes its progression leading to earlier deflection. We noted that, as the first mechanism was activated by the impact 
pressure, it did not modify the slope basis as much. Conversely, the second one dragged wires along and may have strongly modified the material state in some distant areas suggesting non-local effects.

\subsection{Tortuousness and anisotropy of the wire network}

In its current form, TexSol is loaded with small transformations only. These can be split into two types of test with elementary loading: the compression test, which is described in [7], and the shear test. The latter is simulated to highlight the wire network transformation under quasi-static loading. The numerical TexSol sample of $1.6 \mathrm{~cm}$ side, included 8000 particles with 1500 to model a wire with a volume length density of $200 \mathrm{~km} . \mathrm{m}^{-3}$. Particle polydispersity ranged linearly from $\varnothing 0.4 \mathrm{~mm}$ to $\varnothing 1 \mathrm{~mm}$. Global pure shear strain is not easy to obtain with a granular sample. The TexSol is thus wedged in a parallelepiped box. Two parallel lateral planes are applied to rotate at an angular rate of $\dot{\alpha}(t)=\frac{1}{T} \cos ^{2}\left(\tan ^{-1}\left(\frac{t}{T}\right)\right), t \in[0, T]$, where $T$ is the total time to obtain a global shear strain $\gamma=1$, i.e. $\alpha=\frac{\pi}{2}$. These boundary conditions are used to simulate linear variations in the shear strain, i.e. a constant translation of the upper plane. At this stage, two different values are introduced to analyze wire network transformation. Tortuousness, i.e. the state of sinuous objects making several curves, is an important property of a mixed network. We measure it by defining a tortuousness coefficient $\xi=\frac{1}{L} \int_{0}^{L} \varrho^{-1}(s) d s$, where $L$ is the wire length and $\varrho(s)$ is the curvature radius at the curvilinear abscissa $s$. The second interesting value is the anisotropy coefficient $a$, which is a classic parameter to describe contact networks in granular media $[2,12]$. Its expression is given by $a=2 \frac{\mathscr{D}(\boldsymbol{F})}{\operatorname{tr}(\boldsymbol{F})}$ where $\mathscr{D}($.$) is the deflection operator$ such that $\mathscr{D}(\boldsymbol{z})=\left(\sum_{i \neq j}\left(z_{i}-z_{j}\right)^{2}\right)^{0.5}$ (with $z_{i}$ being the principal values of $\boldsymbol{z}$ ) and $\boldsymbol{F}$ is the fabric tensor. Maximum anisotropy is reached for unidirectional structures, i.e having only one non-null principal value of the fabric tensor. The limit value is $a_{\max }=2 \sqrt{2}$. Another remarkable value $a=\sqrt{2}$ is reached for structures with a plane distribution. In continuum mechanics, this shear test is regarded as homogeneous because strains and stresses are the same at any sample point. Unfortunately, this is not as easy in granular media. The rotation axes of the side planes come from the ground, leading to asymmetric loading which strongly influence the contact network (cf. Fig. 4(a)). Grains far from the rotation axes have substanially moved but their contact reactions are negligible compared to those of grains close to rotation axes. Indeed, this sample part is jammed and perpendicularly resists the deformation induced by mobile planes, whereas the remaining particles move quasi-unconstrained. Fig. 4(b) also highlights that the wire network adapts itself to loading by increasing its proportion of tended wires following the principal traction direction. Beyond a stable area, the reinforcement network changes structure. It reorganizes according to the strain because it is dragged by the neighbor- 

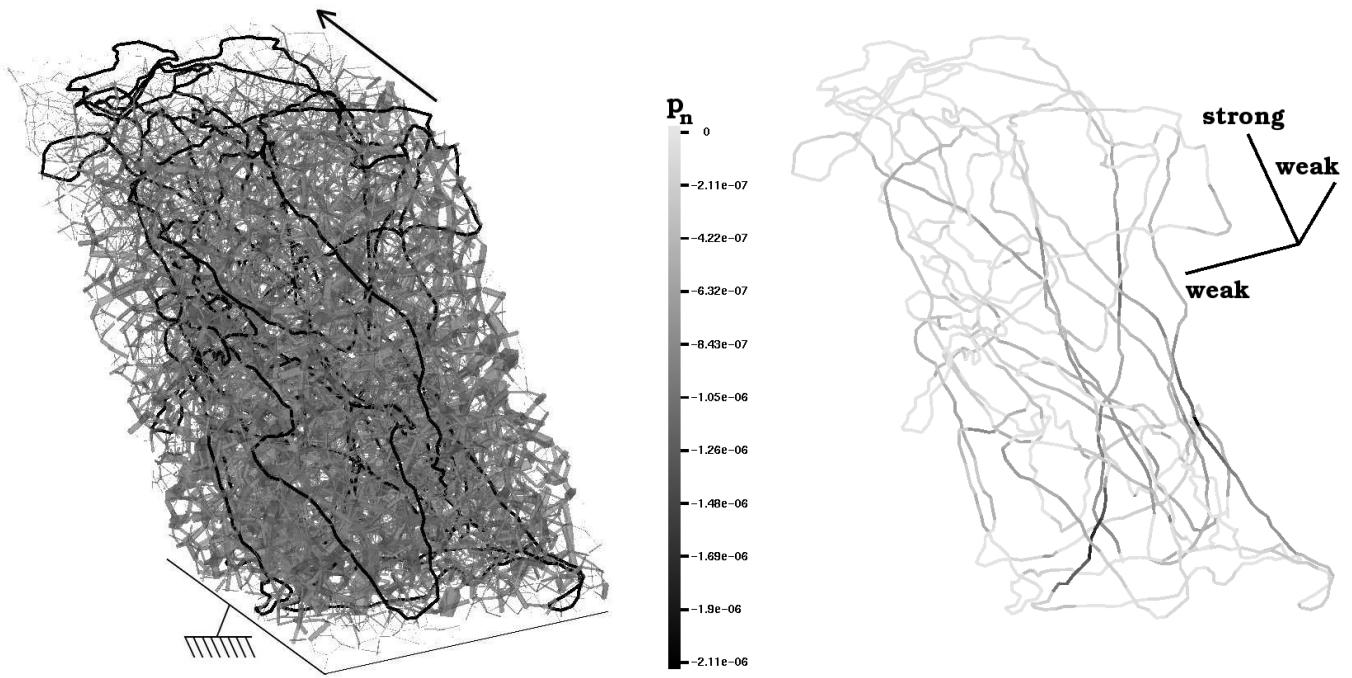

(a) Distributions of the wire network as black lines and contact reactions as grey needles of width proportional to the intensity.

(b) Tensile reactions $p_{n}$ in the deformed wire network and principal basis of the wire stress computed with the Weber tensor $[2]$.

Fig. 4. Contact and wire interaction representations for a shear test. The fixed plan and mobile plane are at the bottom and top of the figures, respectively.

ing particle displacement. This straightens some wire segments, thus lowering
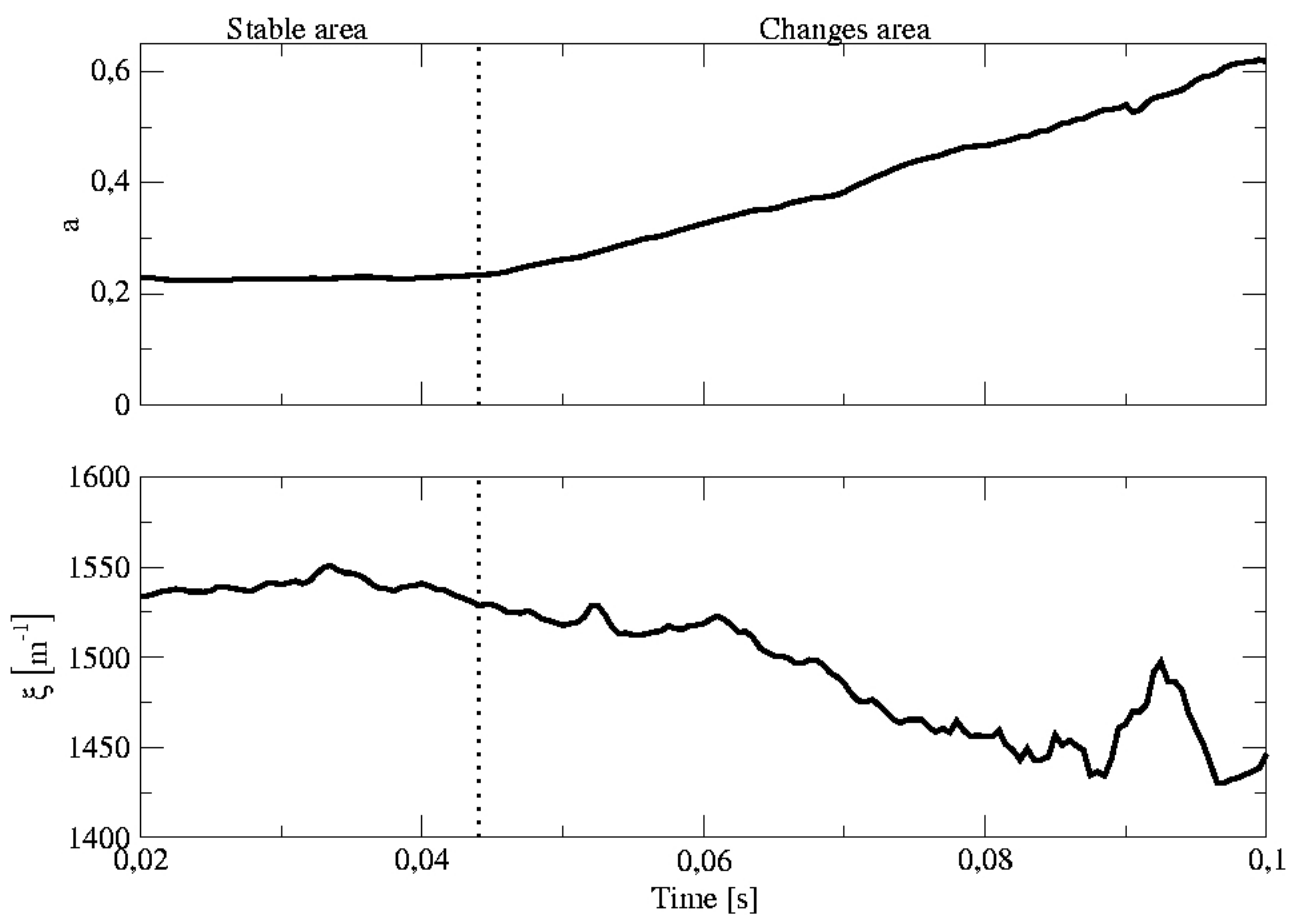

Fig. 5. Time-course plots of tortuousness and anisotropy for a shear test. The boundary between the stable area and the change area is represented by a dotted line.

the tortuousness and increasing anisotropy (cf. Fig. 5). However, this change 
is opposed to the causal motion. In fact, the dominant principal direction of the wire stress tensor (obtained by the Weber tensor [2]) corresponds to the sample lengthening direction as shown in Fig. 4(b).

Two simulations performed in [7] and useful for the rest of the study are recalled to complete our range of TexSol mechanical tests. The first is a quasistatic triaxial test whereby a pressurized granular material is compressed. This test was simulated on a numerical sample close to that of a shear test. The second involved a cylindrical TexSol sample deposited on a rubber surface. The sand part is made of 26000 particles with polydispersity ranging from $\varnothing 0.2 \mathrm{~mm}$ to $\varnothing 0.6 \mathrm{~mm}$. The wire network is composed of 2900 beads of $\varnothing 0.2 \mathrm{~mm}$ diameter with a volume length density of $400 \mathrm{~km} \cdot \mathrm{m}^{-3}$. This test, which is highly dynamic in the transitional phase, leads to large transformations with several changes in the wire network, thus emphasizing non-local effects.

\section{Characteristic length}

TexSol is a material with internal length, i.e. it can generate remote forces and thus behaves like a non-local medium. The coefficient describing the interaction length of the wire is the characteristic length denoted $l_{\mathrm{c}}$. Indeed, in non-local continuum mechanics, the equilibrium of a material point $(x)$ must take the effects of a neighboring domain $\mathcal{D}\left(l_{\mathrm{c}}\right)$ into account[3]. The non-local force due to remote interactions can be written,

$$
F_{\mathrm{nl}}(x)=\int_{\mathcal{D}}(x-y) h(2(x-y) \cdot(U(x)-U(y))) d y,
$$

where $h$ is a fairly smooth function and $U$ is a kinematic variable, i.e. velocities in our case. A non-local behavior identification procedure would involve determining both the $l_{c}$ length and the $f: y \rightarrow 2(x-y) \cdot(U(x)-U(y))$ function. Our study focuses on the characteristic length via a numerical investigation. Remote interactions are obvioulsly transmitted by the wire. Moreover, such tension forces must be strong enough on a continuous part of wire. We then introduce the following definition.

Definition 1 Let $\bar{p}$ be the average tension over the wire network and $m$ a positive coefficient,

$$
{ }^{m} \lambda=\sup _{s_{1}, s_{2}}\left\{\int_{s_{1}}^{s_{2}} d s\left|\forall s \in\left[s_{1}, s_{2}\right],\right| p(s) \mid \geq m \bar{p}\right\}
$$

where $s, s_{1}$ and $s_{2}$ are curvilinear wire coordinates. 
The ${ }^{m} \lambda$ length is the maximum length over which the tension is greater than $m$ times the average tension $\bar{p}$. From a numerical stand point, the tension is constant between two successive beads and the integral is easy to compute. Several parameters $m$ where tested in different mechanical tests, with $m \simeq 3$ providing the most acceptable solution, i.e. dominant wire elements are taken into account but not negligible wire ones. Before making any comparisons, a benchmark test was simulated with a pure non-local load to check ${ }^{3} \lambda$ definition relevancy. A wire extraction test was thus carried out with an imposed vertical velocity of a wire end equal to $0.3 \mathrm{~m} . \mathrm{s}^{-1}$ for $50 \mathrm{~ms}$. In practice, loading is applied on the last bead of the wire, located at the sample top. Changes occurring inside the sample are only due to wire action. During the test, the
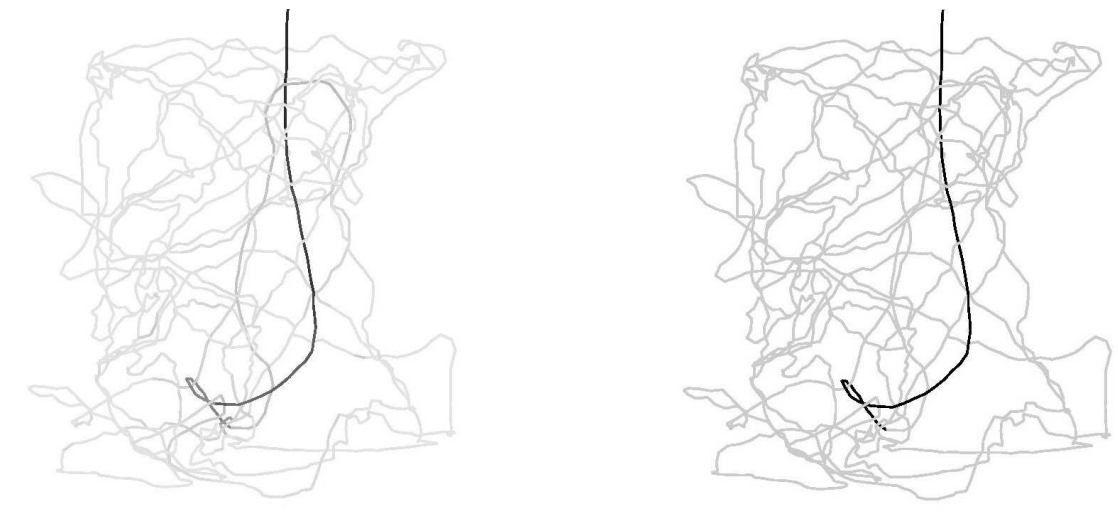

$\mathbf{p}_{\mathbf{n}} \mathbf{i}^{\mathbf{2}}$

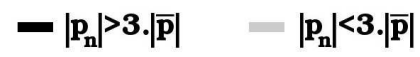

Fig. 6. Comparisons between the wire network tensile distribution $p_{n}$ and the wire segments in action for a wire extraction test. Active wire segments such as $\left|p_{n}\right|$ greater than threefold the average tensile value $|\bar{p}|$, are displayed in black and $l_{\mathrm{c}}=36 \varnothing_{\mathrm{avg}}$.

wire increasingly modifies the granular sample, reaching areas far from the loading source. Indeed, the ${ }^{3} \lambda$ patterns plotted in Fig. 7 shows an increasingly tended wire length. This is associated with the tortuousness plotted in the same figure to identify two phases. The first is the wire binding phase, i.e. the wire segment close to loading is gradually straightened, causing a marked decrease in tortuousness. During this partial straight phase, the maximum tended wire length increases slightly. The wire easily moves sand particles which prevent it from binding until it reaches the jamming state. This situation is caused by the sample bottom pressure which is too high for extraction to continue. This leads to a second more brutal phase which propagates the wire tension beyond many jamming sand configurations. Tortuousness is not really affected by this phase because the wire keeps its general structure while its end is extracted. The maximum tended wire length then reaches very high values corresponding to 36 average particle diameters. At the end of the test, some moving fibers cross and drag other static wire segments and may generate nodes inside the sand. This greatly increases the average tensile state of the wire network. Finally, the ${ }^{3} \lambda$ parameter gives relevant data on remote 

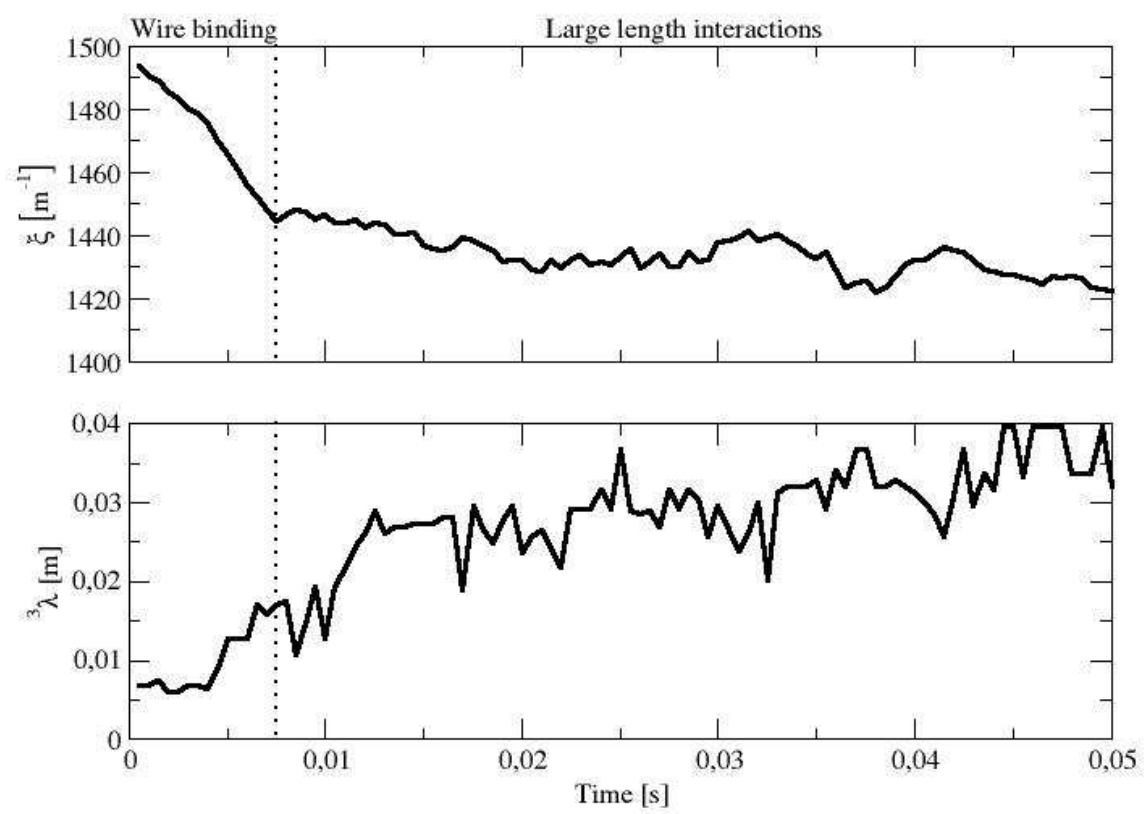

Fig. 7. Time-course variations in tortuousness and ${ }^{3} \lambda$ for a wire extraction test. The wire binding limit is plotted as a dotted line in the two graphs.

interactions, which is thus assimilated to a characteristic length $l_{\mathrm{c}}$. More generally, the ${ }^{3} \lambda$ coefficient (cf. Def. 1) is also highlighted in a pile deposit test, a shear test and a triaxial test in Fig. 8(b), 8(a) and 8(c), respectively. Tests involving large transformations such as pile deposit, and to a lesser extent the shear test, lead to changes in the reinforcement structure, whereby the wire is straightened in the dominant tensile direction to resist better. This mechanism propagates wire tension throughout the sample and increases the remote effects. Conversely, the loading applied in the triaxial test generates small transformations and consequently imposes wire network quasi-staticity. This test involves short active wire elements equiprobably distributed throughout the whole sample. In conclusion, the simulations showed that the remote effects depend only on the loadings. In other words, the internal length cannot be defined as a material characteristic. 

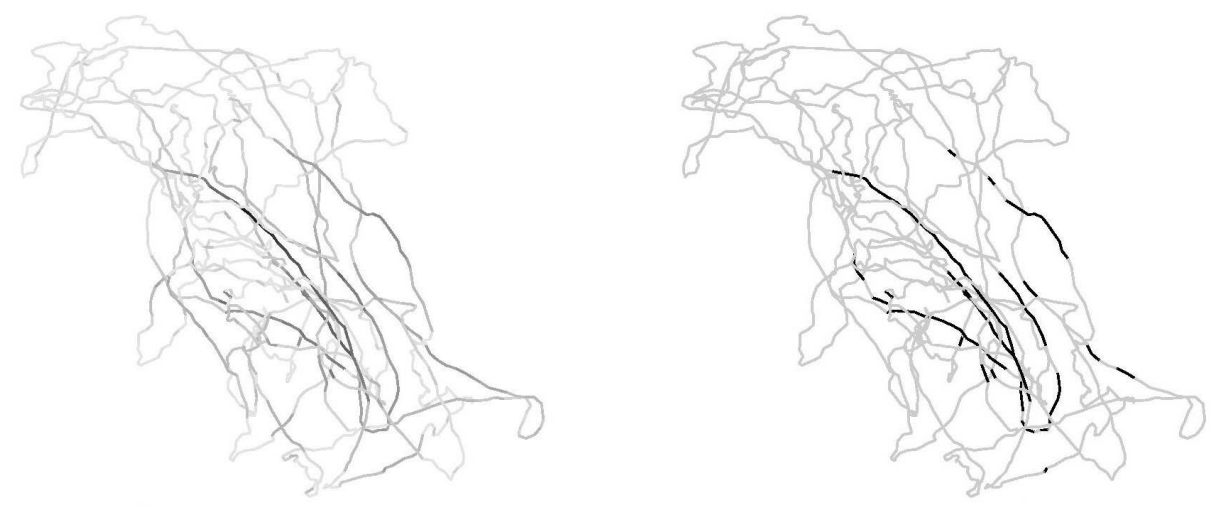

$\mathbf{p}_{\mathbf{n}} \stackrel{0}{!}$

$-\left|\mathbf{p}_{\mathbf{n}}\right|>\mathbf{3} \cdot|\overline{\mathbf{p}}|=\left|\mathbf{p}_{\mathrm{n}}\right|<3 \cdot|\overline{\mathbf{p}}|$

(a) Shear test with $l_{\mathrm{c}}=10 \varnothing_{\mathrm{avg}}$.
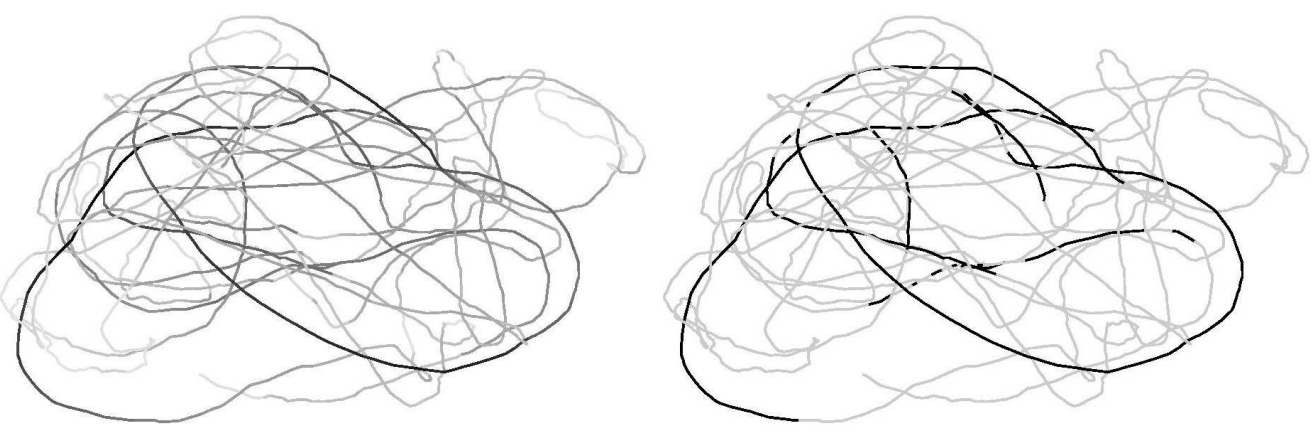

$\mathbf{p}_{\mathbf{n}} \stackrel{0}{\mathbf{1}}$ $-\left|\mathbf{p}_{\mathrm{n}}\right|>\mathbf{3} \cdot|\overline{\mathbf{p}}| \quad-\left|\mathbf{p}_{\mathbf{n}}\right|<3 \cdot|\overline{\mathbf{p}}|$

(b) Pile deposit test with $l_{\mathrm{c}}=54 \varnothing_{\mathrm{avg}}$.
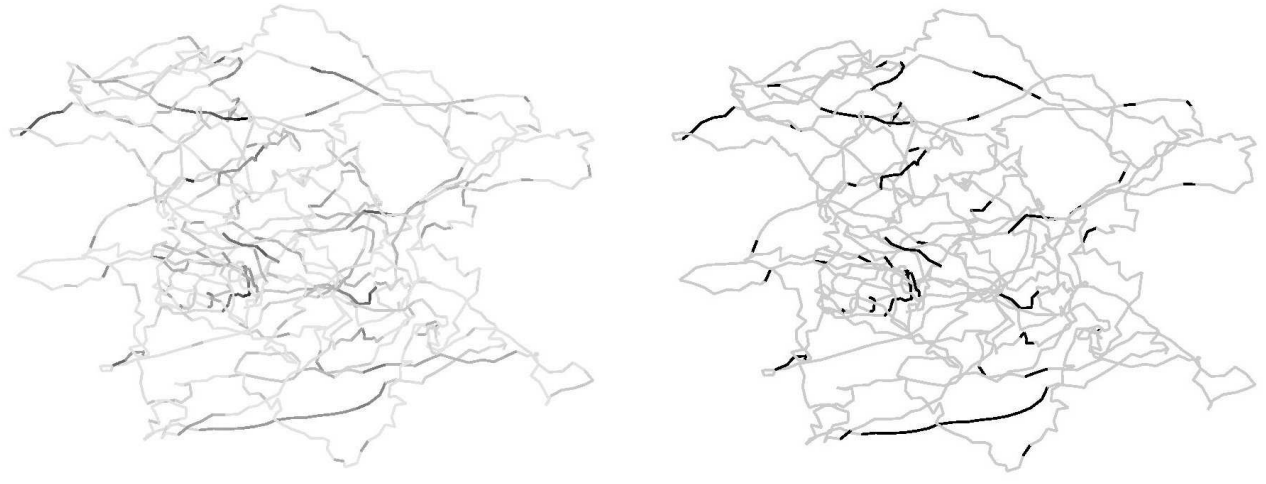

$\mathbf{p}_{\mathbf{n}} \stackrel{0}{\mathbf{1}}$

$\underset{-2.95-06}{-\mathbf{d}}-\left|\mathbf{p}_{\mathrm{n}}\right|>\mathbf{3} \cdot|\overline{\mathbf{p}}| \quad-\left|\mathbf{p}_{\mathbf{n}}\right|^{<3} \cdot|\overline{\mathbf{p}}|$

(c) Triaxial test with $l_{\mathrm{c}}=4 \varnothing_{\text {avg }}$.

Fig. 8. Comparisons between the wire network tensile distribution $p_{n}$ and wire segments in action for different mechanical tests. Active wire segments such as $\left|p_{n}\right|$ greater than threefold the average tensile value $|\bar{p}|$, are displayed in black. 


\section{From discrete to continuous: an identification procedure}

\subsection{A continuous model}

At the macroscopic scale, TexSol can be described by models using local or non-local formalism (cf. [14, 3]). In [6], in a small strain domain, we propose a thermodynamically consistent TexSol model described by two potentials: free energy $\psi=\psi_{s}+\psi_{w}$, where ${ }_{s}$ and ${ }_{w}$ index the sand phase and wire network phase, respectively, along with the dissipation potential $\varphi$. A sand elastic-plastic model and a wire unilateral elastic model are thus overlapped on the basis of two assumptions: stress additivity $\boldsymbol{\sigma}=\boldsymbol{\sigma}_{s}+\boldsymbol{\sigma}_{w}$ and strain rates equalities $\dot{\varepsilon}=\dot{\varepsilon}_{s}=\dot{\varepsilon}_{w}$. The numerical experiments referred to in [7] give the limits of these assumptions which remain valid as long as the wire network does not slide with respect to the sand matrix. The state variable is chosen as the total strain $\boldsymbol{\varepsilon}$ and is combined with internal variables $\boldsymbol{\varepsilon}^{\mathrm{p}}, \boldsymbol{\alpha}$ and $p$ describing the plasticity, kinematic and isotropic hardening, respectively. Elastic parameters are the Hooke tensor $\mathbb{K}_{s}$ and Lamé coefficients $\lambda_{w}$ and $\mu_{w}$; hardening parameters are $H_{\mathrm{i}}$ and $H_{\mathrm{k}}$, i.e. the isotropic and kinematic hardening, respectively. Single-phase free energies are written as,

$$
\begin{aligned}
& \psi_{s}\left(\varepsilon, \varepsilon^{\mathrm{p}}, \boldsymbol{\alpha}, p\right)=\frac{1}{2}\left(\varepsilon-\varepsilon^{\mathrm{p}}\right): \mathbb{K}_{s}\left(\varepsilon-\varepsilon^{\mathrm{p}}\right)+\frac{H_{\mathrm{k}}}{2} \boldsymbol{\alpha}: \boldsymbol{\alpha}+\frac{H_{\mathrm{i}}}{2} p^{2} \\
& \psi_{w}(\varepsilon)=\frac{\lambda_{w}}{2}\langle\operatorname{tr}(\varepsilon)\rangle^{2}+\mu_{w} \varepsilon^{\geq}: \varepsilon^{\geq},
\end{aligned}
$$

where $\langle\cdot\rangle=\max (\cdot, 0)$. The unilateral operator, denoted $: \geq$, is defined by $\varepsilon^{\geq}=$ $\boldsymbol{P}\left\langle\operatorname{diag}\left(\varepsilon_{1}, \varepsilon_{2}, \varepsilon_{3}\right)\right\rangle \boldsymbol{P}^{T}$, where $\varepsilon_{1}, \varepsilon_{2}, \varepsilon_{3}$ represents the principle values and $\boldsymbol{P}$ is the transformation matrix from the principal directions to the current ones. The wire network model is non-dissipative, so the TexSol and sand dissipation potentials are the same. We thus write the Legendre - Fenchel transform of the dissipation potential as,

$$
\varphi^{*}\left(\boldsymbol{\sigma}^{i r}, \boldsymbol{A}, \boldsymbol{\chi}, R\right)=\mathrm{I}_{\{\mathbf{0}\}}\left(\boldsymbol{\sigma}_{s}^{i r}\right)+\mathrm{I}_{\Omega(\boldsymbol{\chi}, R)}(\boldsymbol{A}),
$$

where $\boldsymbol{\sigma}^{\text {ir }}, \boldsymbol{A}, \boldsymbol{\chi}$ and $R$ are thermodynamic forces associated with $\boldsymbol{\varepsilon}, \boldsymbol{\varepsilon}^{p}, \boldsymbol{\alpha}$ and $p$, respectively. The indicator function of a set $\mathcal{D}$ is denoted $\mathrm{I}_{\mathcal{D}}(\cdot)$. In the principal stress space, $\Omega$ represents the elastic domain which, in our granular case, is bounded by a Drucker - Prager criterion depending on the internal friction parameter $\beta$ and the initial cohesion $C_{0}$. These potentials are convex $[6,15]$ and minimum at the origin, so they verify the $1^{\text {st }}$ and $2^{\text {nd }}$ thermodynamic principles a priori. The TexSol behavior laws deriving from $\psi$ and $\varphi$, are implemented in the finite element software Cast $3 M^{\mathrm{TM}}$. To connect microscopic (granularity, friction, volume length density of wire, et catera) with 
macroscopic parameters (elasticities, hardening, et catera), a homogenization procedure may be carried out whereby continuous and discrete numerical models are associated with a backward analysis. The lack of investigation and micro-parameter measurements deriving from classical experiments, warrants the use of value fields computed with a discrete numerical model. However, because of the high number of parameters $\Theta=\left\{E_{s}, \nu_{s}, E_{w}, \nu_{w}, C_{0}, \beta, H_{\mathrm{k}}, H_{\mathrm{i}}\right\}$ describing the TexSol continuous model, a single set of parameters is identified through a homogeneous mechanical test; the triaxial test is well tailored for such a simulations. Numerical fields deriving from the simulations are averaged over the whole sample. This approach may be related to Finite Element Method Updating.

\subsection{Simple identification framework}

The task is to identify the mechanical parameters of two phases, i.e. sand and wire, starting from a simulation of the mixture. The simulations allow separate computation of stress and strain fields associated with the sand and the wire network, respectively. The equality of the strain fields $\varepsilon_{s}$ and $\varepsilon_{w}$ can be verified. The identification procedure consists of three steps by splitting the parameter set into three subsets:

- $\Theta_{\mathrm{e}}=\left\{E_{s}, \nu_{s}, E_{w}, \nu_{w}\right\}$ for purely elastic coefficients,

- $\Theta_{y}=\left\{\beta, C_{0}\right\}$ for elastic yield, and

- $\Theta_{\mathrm{p}}=\left\{H_{\mathrm{k}}, H_{\mathrm{i}}\right\}$ for plastic hardening.

In the first step, elastic coefficients are identified in a simple tension/compression test restricted to the elastic domain. The equality of the strain fields leads us to consider the same Poisson coefficient for the sand and wire network,

$$
\boldsymbol{\varepsilon}=\boldsymbol{\varepsilon}_{w}=\boldsymbol{\varepsilon}_{s}=\left[\begin{array}{ccc}
\varepsilon & 0 & 0 \\
0 & -\nu \varepsilon & 0 \\
0 & 0 & -\nu \varepsilon
\end{array}\right]
$$

where $\varepsilon$ is the imposed strain and $\nu=\nu_{s}=\nu_{w}$ the apparent Poisson coefficient. The confinement pressure in a homogeneous triaxial test does not modify the form of the strain. From the TexSol continuous model (cf. Eq. (4) and (5)), 
stresses are written as,

$$
\begin{aligned}
\boldsymbol{\sigma}_{s} & =\lambda_{s}(1-2 \nu) \varepsilon \boldsymbol{I}+2 \mu_{s} \operatorname{diag}(\varepsilon,-\nu \varepsilon,-\nu \varepsilon)=\left[\begin{array}{ccc}
E_{s} \varepsilon & 0 & 0 \\
0 & 0 & 0 \\
0 & 0 & 0
\end{array}\right], \\
\boldsymbol{\sigma}_{w} & =\lambda_{w}(1-2 \nu)\langle\varepsilon\rangle \boldsymbol{I}+2 \mu_{w} \operatorname{diag}(\langle\varepsilon\rangle,\langle-\nu \varepsilon\rangle,\langle-\nu \varepsilon\rangle) \\
& =\lambda_{w}(1-2 \nu)\langle\varepsilon\rangle \boldsymbol{I}+2 \mu_{w} \operatorname{diag}(\langle\varepsilon\rangle,-\nu\rangle \varepsilon\langle,-\nu\rangle \varepsilon\langle) \\
& =\left[\begin{array}{ccc}
E_{w}\langle\varepsilon\rangle & 0 & 0 \\
0 & \frac{E_{w} \nu}{1+\nu}|\varepsilon| & 0 \\
0 & 0 & \frac{E_{w} \nu}{1+\nu}|\varepsilon|
\end{array}\right]
\end{aligned}
$$

where $\rangle \cdot\left\langle=\min (\cdot, 0)\right.$. In any case, the identification of $E_{s}$ is obvious. The Young modulus $E_{w}$ can be determined from the tensile direction or from the transverse directions in tension or compression state. In the latter case, we chose the average value in order to minimize measurement error,

$$
\begin{aligned}
& \nu=\frac{-\varepsilon_{22}-\varepsilon_{33}}{2 \varepsilon_{11}}, E_{s}=\frac{\sigma_{s, 11}}{\varepsilon_{11}} \\
& \text { and } E_{w}=\left\{\begin{array}{ll}
\frac{1}{2}\left[\sigma_{w, 22}\left(\frac{1}{\varepsilon_{11}}+\frac{1}{\varepsilon_{22}}\right)+\sigma_{w, 33}\left(\frac{1}{\varepsilon_{11}}+\frac{1}{\varepsilon_{33}}\right)\right] & \text { if } \varepsilon_{11}>0 \\
\frac{1}{2}\left[-\sigma_{w, 22}\left(\frac{1}{\varepsilon_{11}}+\frac{1}{\varepsilon_{22}}\right)-\sigma_{w, 33}\left(\frac{1}{\varepsilon_{11}}+\frac{1}{\varepsilon_{33}}\right)\right] & \text { if } \varepsilon_{11} \leq 0
\end{array}\right. \text {. }
\end{aligned}
$$

In the second step, compression tests with different confinement pressures are performed to identify the elastic yield depending on a cohesion coefficient. Thirdly, the hardening parameters were identified separately by Finite Element Method Updating limited to hardening, i.e. knowing the six other parameters. The cost function to minimize is the difference between the plastic slope of numerical discrete experiments and that of continuous modeling.

\subsection{Identification results}

Identification is carried out using numerical granular TexSol samples of that are greater in size than a representative volume. This depends on the characteristic length which must be quite short. According to Fig. 8, a triaxial compression test is recommended. Elastic identification requires extraction of a reversible domain of $\left(\boldsymbol{\varepsilon}_{s}, \boldsymbol{\sigma}_{s}\right)[1,2]$. The difficulty is to determine the elastic parameters of a macro-model starting from the simulation of a granular 
material modeled as a collection of rigid bodies in contact. Indeed, material deformation is obtained by micro-slips. We speak of apparent elasticity when these micro-slips vanish at the end of a loading cycle. Moreover, the initial equivalent stress $\sigma_{s}^{\text {eq }}$ has to be recovered. The numerical samples mentioned in the shear test (cf. Sect. 2.2) are pressurized under 0.5, 0.6, 0.7, 0.8, 0.9 and 1 bar in a parallelepipedic box. A cyclic triaxial load is imposed by the upper bound displacement according to a triangular signal where its maximum increases each cycle (MM). To determine the apparent elastic cycles, the equiv-

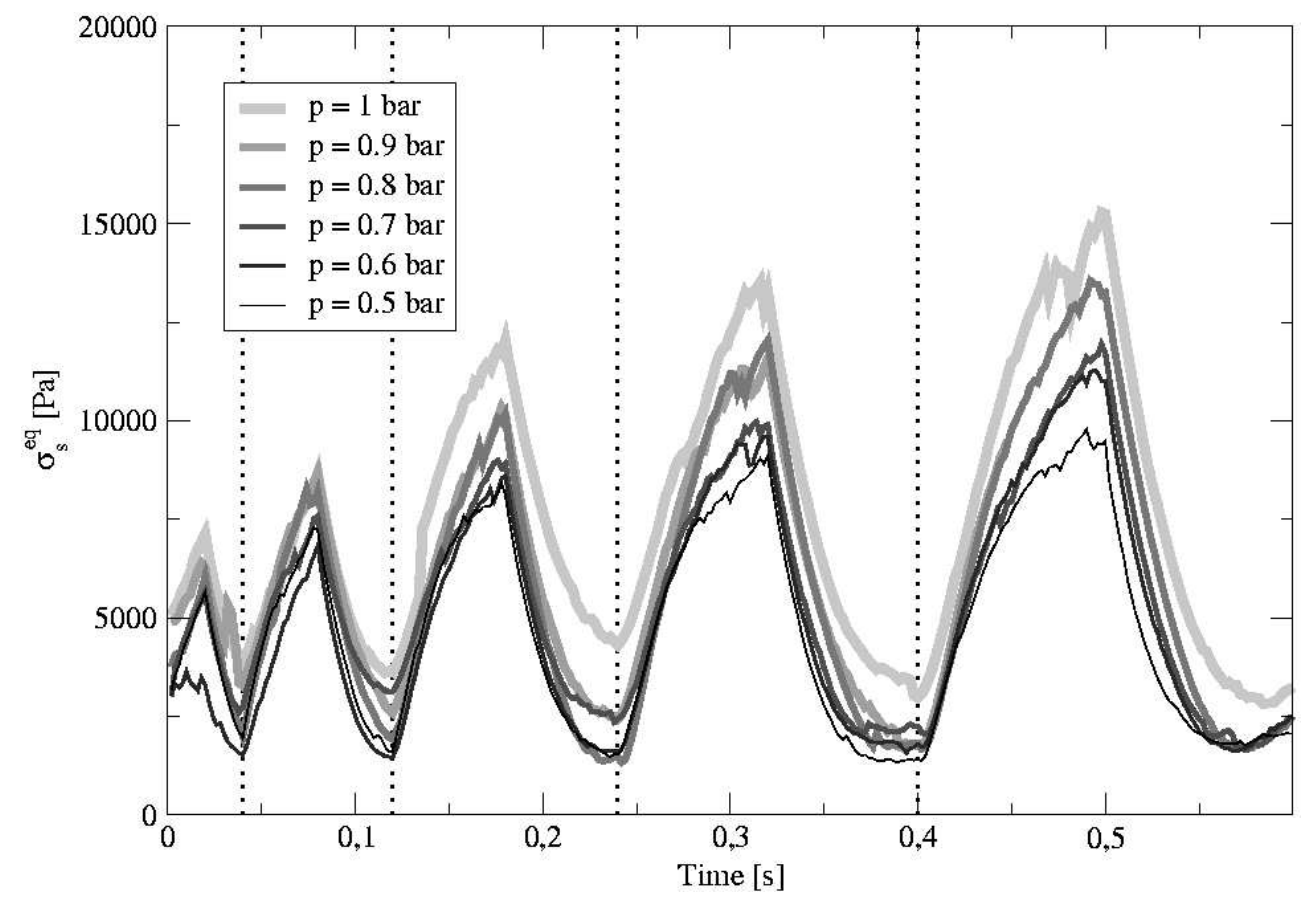

Fig. 9. Evolution of $\sigma_{s}^{\text {eq }}$ for several cyclic triaxial tests, where $\sigma_{s}^{\text {eq }}=\sqrt{J_{2}\left(\boldsymbol{S}_{s}\right)}$ is the stress deviatoric norm and $p=-\frac{1}{3} \operatorname{tr}\left(\boldsymbol{\sigma}_{s}\right)$ is the confinement pressure.

alent stress $\sigma_{s}^{\text {eq }}$ for the sand is plotted over a time-course in Fig. 9. At the end of the first three cycles, $\sigma_{s}^{\text {eq }}$ decreases almost linearly until reaching the initial value. A contrario, for the two last cycles, the equivalent stress decreases to a value less than the initial one before the end of the cycle. The first three cycles thus satisfy the apparent elasticity notion. In Fig. 10, two estimations tend to two different constant values expressing a sample anisotropy. To identify an isotropic model considered in the previous section, we chose the average, as given in Eq. $(9)^{1}, \nu=0.25$. The Young moduli are extracted from the stress vs. strain relationship (e.g. in Fig. 11 and 12) according to Eq. $(9)^{2}$. Once $\Theta_{\mathrm{e}}$ is known, elastic yield parameters are identified by recording the stress deviatoric intensity when the real behavior becomes incompatible with the elastic prediction. From the initial pressurized samples, triaxial tests equivalent to the last cycle are simulated and the linear regression of the sand elastic yield points gives a Drucker - Prager envelop approximation where $C_{0}=18810 \mathrm{~Pa}$ and $\beta=0.067$ which is close to the maximum shear pattern of the second 


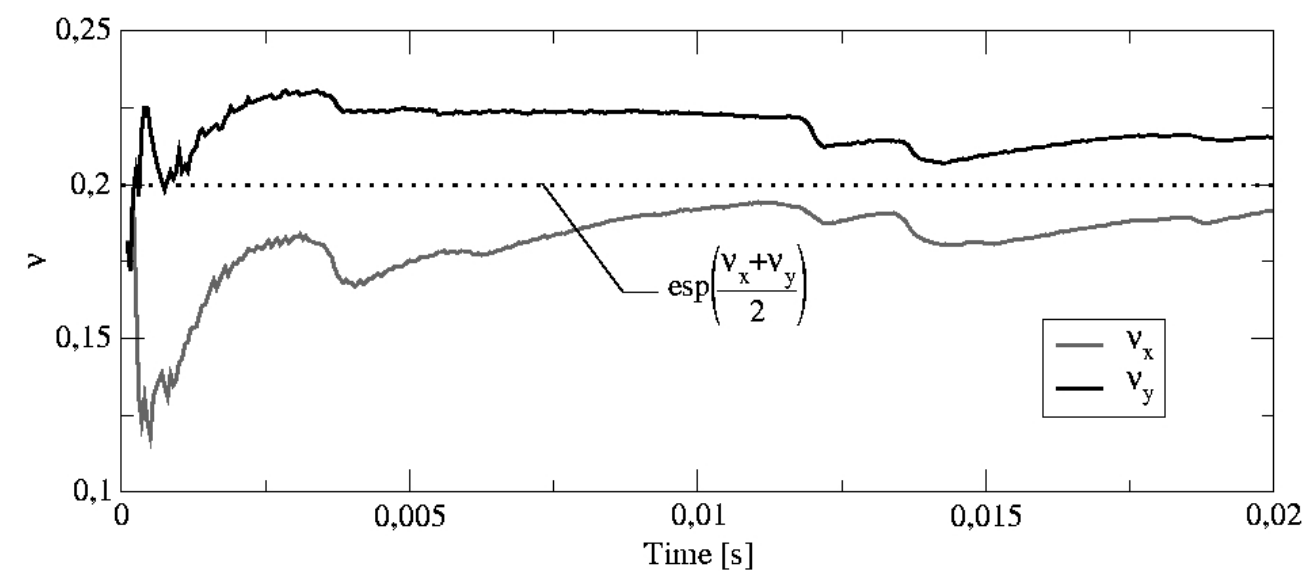

Fig. 10. Time-course variations in the two estimations of the Poisson coefficient, $\nu_{x}=-\frac{\varepsilon_{x x}}{\varepsilon_{z z}}$ and $\nu_{y}=-\frac{\varepsilon_{y y}}{\varepsilon_{z z}}$ for the first cycle. The average values of these two estimations is displayed as a dotted line.

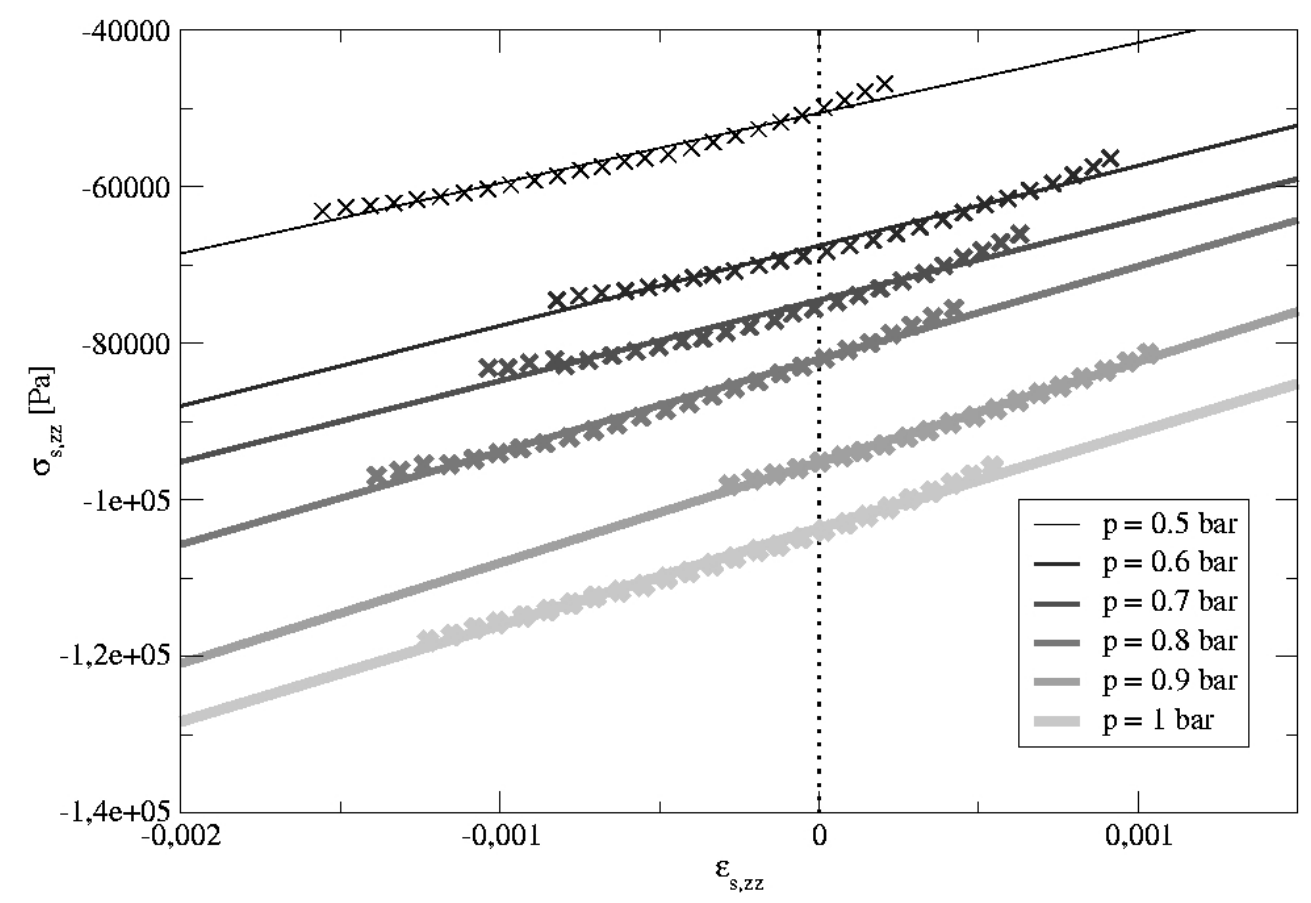

Fig. 11. Sand stress vs. sand strain experimental points $\left(\varepsilon_{s, z z}, \sigma_{s, z z}\right)$ derived from the various discrete simulations in the elastic domain. Their linear regression is also displayed as a continuous line of the same color (for the third cycle).

cycle (cf. Fig. 13). Note that $\Theta_{y}$ identification indicates an initial cohesion of the sand phase resulting from the wire network reaction to the TexSol sample preparation process. For the plastic parameters, as only triaxial data is available, the proportion of kinematics and isotropic hardening cannot be known. Consequently, each hardening coefficient is independently updated by FEMU according to plastic variation slopes. A ratio of these two values is then updated. This simple identification shows a dependence between the Young modulus, hardening coefficient, and the confinement pressure. Indeed, this is 


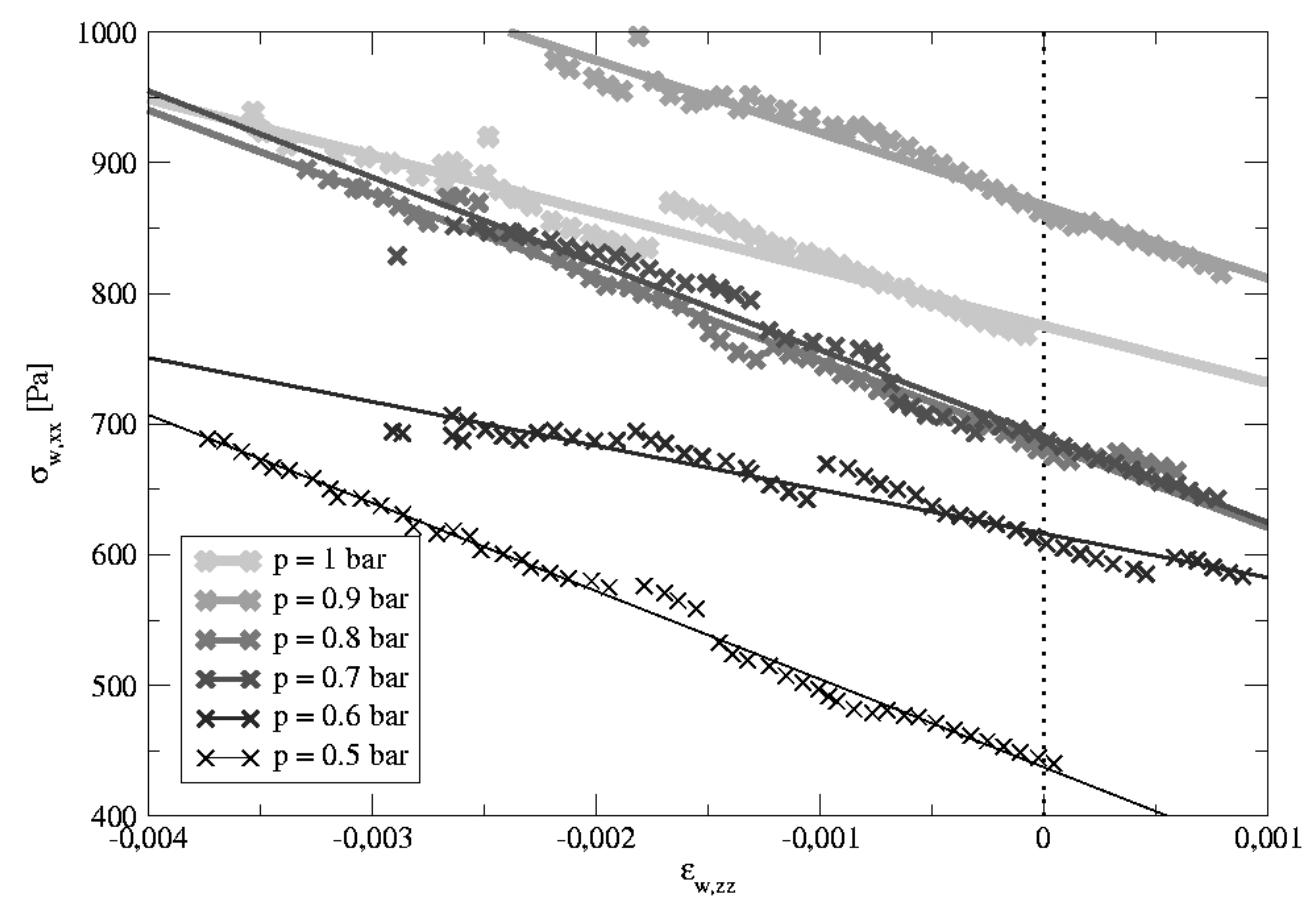

Fig. 12. Wire stress vs. wire strain experimental points $\left(\varepsilon_{w, z z}, \sigma_{w, x x}\right)$ derived from the various discrete simulations in the elastic domain. Their linear regression is also displayed as a continuous line of the same color (for the third cycle).

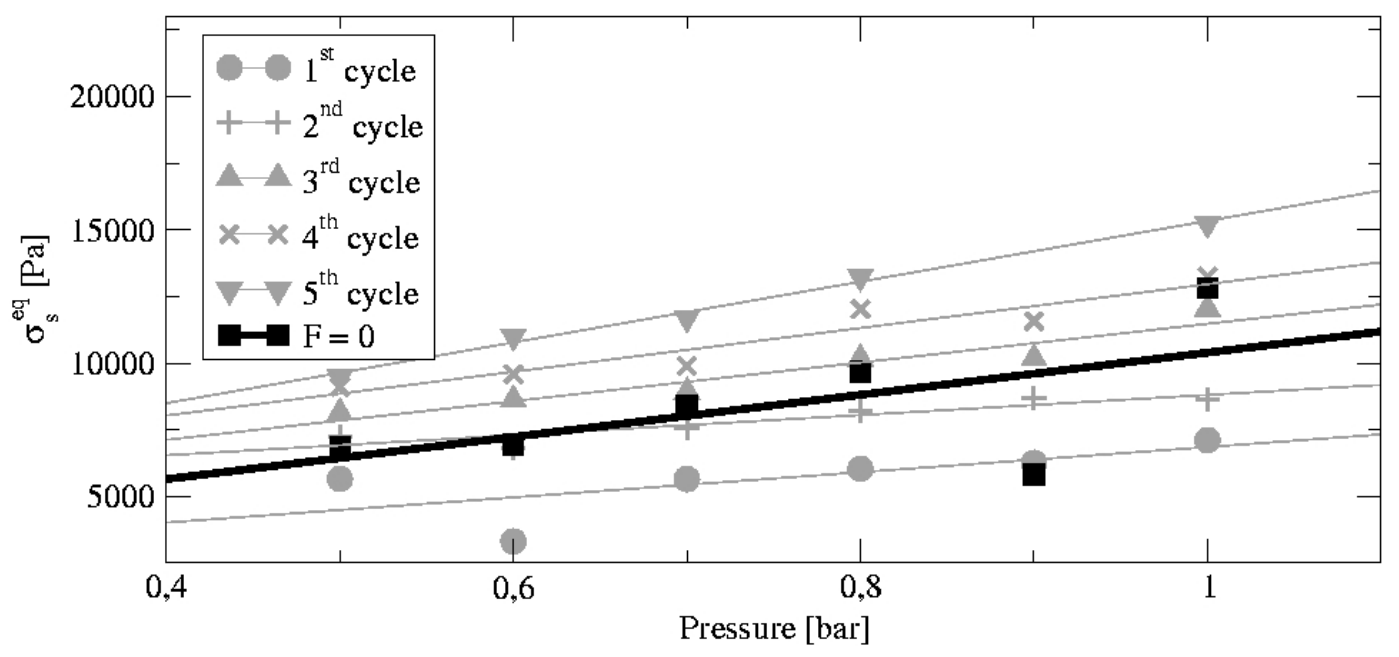

Fig. 13. Shear elastic yield points and regression (displayed in black) according to the pressure for a discrete TexSol sample, where $F=0$ is the load surface. As a reference mark, maximum shear limits and the regression of each cycle are displayed in grey.

partially due to some edge effects supported by high pressure and an angular container. Initial stresses due to the preparation process also cause disturbance. We propose to limit these phenomena by using a cylindrical container and a low confinement pressure range. This pressurization step is carried out via a special procedure [13] which applies precomputed forces directed toward 
the cylinder axis on sample particles located in a thin peripheral layer. To be completely free of edge effects, the identification procedure is limited to a spherical volume included in the sample. Using the same methods as those de-

\begin{tabular}{||c|ccccc||}
\hline \hline Pressure [bars] & $E_{w}[\mathrm{kPa}]$ & $E_{s}[\mathrm{kPa}]$ & $\tau_{y}[\mathrm{~Pa}]$ & $H_{\mathrm{k}}[\mathrm{kPa}]$ & $H_{\mathrm{i}}[\mathrm{kPa}]$ \\
\hline 0.5 & 405.4 & 11302 & 6832 & 800 & 400 \\
0.6 & 321.4 & 12280 & 6947 & 1000 & 500 \\
0.7 & 305.1 & 9701 & 8429 & 1040 & 520 \\
0.8 & 309.5 & 14606 & 9679 & 960 & 480 \\
0.9 & 318.7 & 12948 & 5810 & 920 & 460 \\
1 & 271,4 & 21709 & 12803 & 860 & 430 \\
\hline \hline
\end{tabular}

Table 1

Identification of elastic modulus $E_{s}$ and $E_{w}$, shear limit $\tau_{y}$ and hardening modulus $H_{\mathrm{k}}$ and $H_{\mathrm{i}}$, with respect to the confinement pressure.

veloped in Sect. 4.2 and implemented previously, the continuous TexSol model parameters were identified, as presented in Tab. 1 and $\nu=0.2, C_{0}=2842 \mathrm{~Pa}$ and $\beta=0.079$. Excluding the identified 1 bar-pressurized TexSol value, the elastic modulus and hardening coefficients oscillate around a constant position with a reasonable standard deviation. Removing the edge effect seems to have a stabilizing effect on these coefficients.

\subsection{Validation}

Validation is performed in a non-homogeneous test using discrete and continuous modeling. The numerical TexSol sample used is that of the shear test in a $38 \mathrm{~mm}$ and $\varnothing 29 \mathrm{~mm}$ height cylindrical container with under 2 bars pressure. Its microscopic parameters (granulometry, wire density et ccetera) and macroscopic parameters $(\Theta)$ are known. The test considered is triaxial compression with embedding boundary conditions on the lower and upper faces. Qualitatively, there are enough contact reactions between sand particles to give a homogeneous stress map, in close agreement with that of the continuous model. This is not the case for the wire network (cf. Fig. 14) in our example, which makes comparison harder. Nevertheless, the stress intensity found in the wire network is almost equivalent between the two approaches, particularly at the sample center. The bottom of the discrete wire stress map is also closer to the continuous distribution. The deviatoric stress intensity profile presents a defect at the top. This could be explained by the purely dynamic feature of the software based on the Non-Smooth Contact Dynamics between rigid bodies. It is therefore hard to apply displacement boundary conditions without strongly and locally upsetting the force network close to 


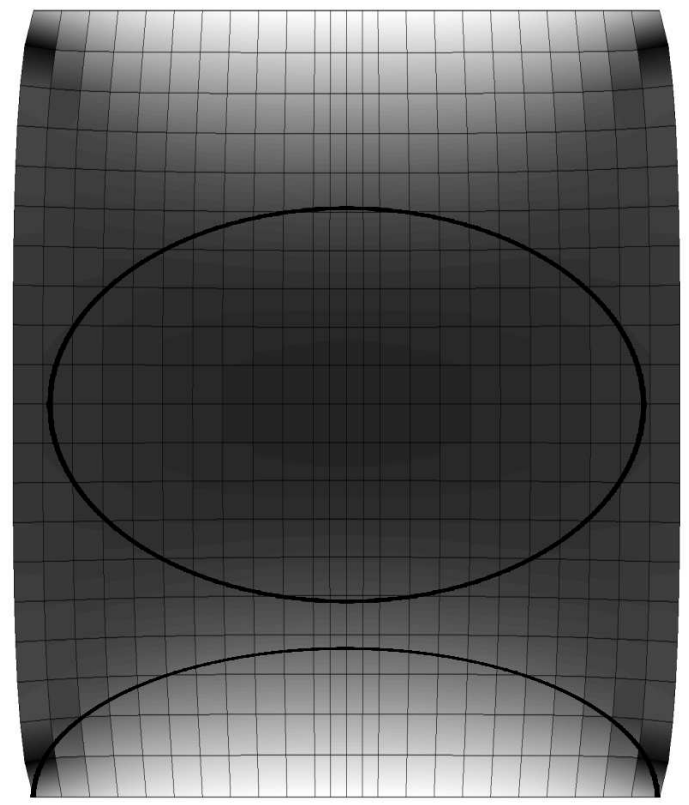

$0 \mathrm{kPa}$

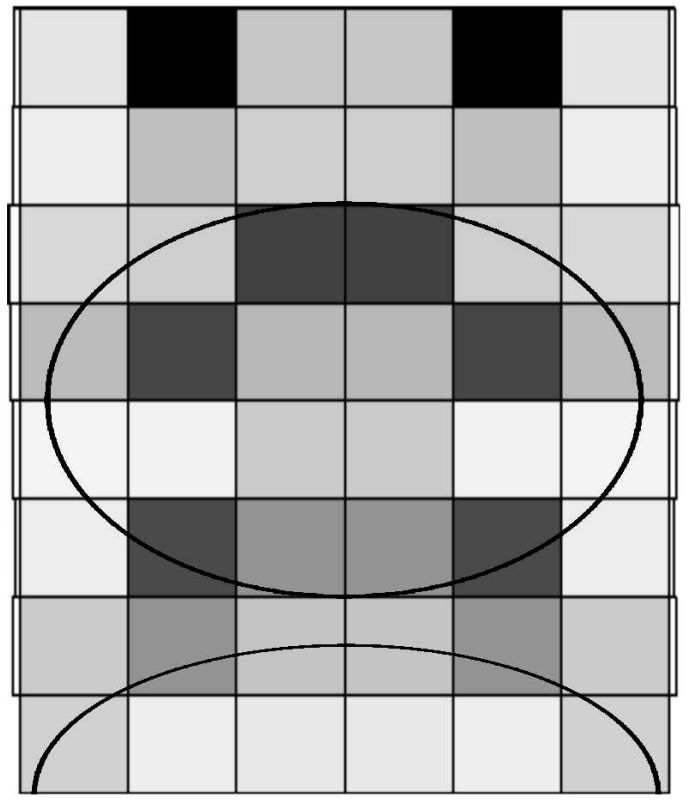

$38 \mathrm{kPa}$

Fig. 14. Comparison of wire deviatoric stress intensity maps in a non-homogeneous triaxial test to compare numerical discrete (right) and continuous (left) models. The discrete map is computed by averaging the tensile reactions on torus fields.

the considered boundary. It is obvious that, when using a larger-size sample with greater computing powers the average torus field would include more wire elements and thus give a more relevant wire stress map.

\section{Conclusions}

Numerical investigations, based on Non-Smooth Contact Dynamics, highlighted the difficulty to propose a unique continuous model for TexSol material. Indeed they revealed different mechanisms according to the loading level. Non-local phenomena, i.e. long distance interactions, appear via a wire network reorganization, generated by large transformations. On the other hand, small transformations allow wire network reinforcement to be seen as a local phenomenon. At the structure scale, unilateral interaction laws produce quite unique behaviors, favoring tensile directions. The discrete model is compared, via numerical homogenization procedures, to a continuous thermodynamic consistent TexSol model. An initial global identification procedure is proposed using numerical fields and generated encouraging results concerning the wire network. However, the homogenization process needs to be improved using FEMU with material parameters roughly identified on larger-size samples. 


\section{References}

[1] B. Cambou, M. Chaze, and F. Dedecker. Change of scale in granular materials. European Journal of Mechanics - A/Solids, (19):999-1014, 2000.

[2] B. Cambou and M. Jean. Micromécanique des matériaux granulaires. Hermès, Science-Paris, 2001.

[3] M. Fremond. Non-Smooth Thermo-mechanics. Springer-Verlag, Berlin Heidelberg New York, 2002.

[4] M. Jean. The non smooth contact dynamics method. Computer Methods in Applied Mechanic and Engineering, (177 (Special issue)):235-257, 1999.

[5] M. Khay and J-P. Gigan. Texsol - ouvrage de soutènement. Technical report, LCPC, 1990.

[6] R. Laniel, P. Alart, and S. Pagano. Consistent thermodynamic modelling of wire-reinforced materials. European Journal of Mechanics - A/Solids, 26:854-871, 2007.

[7] R. Laniel, P. Alart, and S. Pagano. Discrete element investigations of wire-reinforced geomaterial in a three-dimensional modeling. Computational Mechanics, 42(1):67-76, 2007.

[8] R. Laniel, O. Mouraille, S. Pagano, F. Dubois, and P. Alart. Numerical modelling of reinforced geomaterials by wires using the non smooth contact dynamics. In P. Wriggers and U. Nackenhorst, editors, Analysis and Simulation of Contact Problems, pages 289-296. Springer, 2006.

[9] E. Leflaive, M. Khay, and J-C. Blivet. Un nouveau matériaux: le texsol. Travaux, (602):1-3, 1985.

[10] J-J. Moreau. Unilateral contact and dry friction in finite freedom dynamics. In J-J. Moreau and P-D. Panagiotopoulos, editors, Non Smooth Mechanics and Applications, CISM Courses and Lectures, volume 302, pages 1-82. Springer, 1998.

[11] J-J. Moreau. Numerical dynamics of granular materials. In Proceedings of the 3 Contact International Symposium, Peniche (Portugal), pages 1-16. Kmuwer Academics Publishers, June 2001.

[12] F. Radjaï, D. E. Wolf, M. Jean, and J-J. Moreau. Bimodal character of stress transmission in granular packings. Phys. Rev. Lett., 80(1):61-64, 1998.

[13] A. Taboada, K-J. Chang, F. Radjaï, and F. Bouchette. Rheology, force transmission, and shear instabilities in frictional granular media from biaxial numerical tests using the contact dynamics method. Journal of Geophysical Research, 110:1-24, 2005.

[14] P. Villard and P. Jouve. Behavior of granular materials reinforced by continuous threads. Computers and Geothechnics, 7:83-98, 1989.

[15] WH. Yang. A generalized von mises criterion for yield and fracture. $J$. Applied Mechanics, (47):297-300, 1980. 